\title{
New developments in tuberculosis diagnosis and treatment
}

\author{
Cara M. Gill (1), Lorraine Dolan, Laura M. Piggott and Anne Marie McLaughlin
}

Dept of Respiratory Medicine, Saint James's Hospital, Dublin, Ireland.

Corresponding author: Cara M. Gill (cagill@tcd.ie)

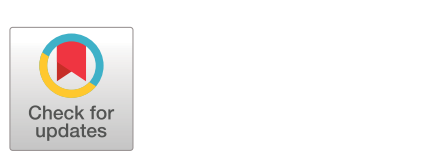

Shareable abstract (@ERSpublications)

Early detection of drug resistance is essential to our goal of global eradication of TB. Tolerable drugs and shorter regimens promote patient adherence. Treating TB infection in vulnerable groups will prevent further global spread of TB disease. https://bit.ly/3oUWOSN

Cite this article as: Gill CM, Dolan L, Piggott LM, et al. New developments in tuberculosis diagnosis and treatment. Breathe 2022; 18: 210149 [DOI: 10.1183/20734735.0149-2021].
Copyright OERS 2022

Breathe articles are open access and distributed under the terms of the Creative Commons Attribution Non-Commercial Licence 4.0.

Received: 1 Oct 2021 Accepted: 16 Nov 2021

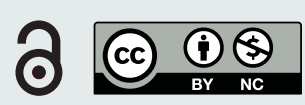

\section{Abstract}

Tuberculosis (TB) is a major cause of morbidity and mortality worldwide. It is estimated that $25 \%$ of the world's population are infected with Mycobacterium tuberculosis, with a 5-10\% lifetime risk of progression into TB disease. Early recognition of TB disease and prompt detection of drug resistance are essential to halting its global burden. Culture, direct microscopy, biomolecular tests and whole genome sequencing are approved methods of diagnosis; however, their widespread use is often curtailed owing to costs, local resources, time constraints and operator efficiency. Methods of optimising these diagnostics, in addition to developing novel techniques, are under review. The selection of an appropriate drug regimen is dependent on the susceptibility pattern of the isolate detected. At present, there are 16 new drugs under evaluation for TB treatment in phase I or II clinical trials, with an additional 22 drugs in preclinical stages. Alongside the development of these new drugs, most of which are oral medications, new shorter regimes are under evaluation. The aim of these shorter regimens is to encourage patient adherence, and prevent relapse or the evolution of further drug resistance. Screening for TB infection, especially in vulnerable populations, provides an opportunity for intervention prior to progression towards infectious TB disease. New regimens are currently under evaluation to assess the efficacy of shorter durations of treatment in this population. In addition, there is extensive research into the use of post-exposure vaccinations in this cohort. Worldwide collaboration and sharing of expertise are essential to our ultimate aim of global eradication of TB disease.

\section{Educational aims}

- Differentiate between TB infection and TB disease.

- Understand the different methods of diagnosing TB disease and resistance.

- Recognise the different drugs and regimens currently in use for TB disease.

- Be able to discuss risk of TB disease in TB infection, and assist patients in making an informed decision on treatment for TB infection.

\section{Introduction}

Tuberculosis (TB) is a major cause of morbidity and mortality worldwide. TB is caused by the bacillus Mycobacterium tuberculosis (Mtb), which is spread via airborne droplets. Approximately one in four people worldwide demonstrate an immunological response to $M t b$ infection, which can remain dormant or progress into active disease forms [1]. Patients infected with TB who have no active signs or symptoms of disease were previously deemed to have latent TB, more recently changed to TB infection [2]. Whereas patients with active disease are termed to have TB disease. Patients with TB infection have a 5-10\% lifetime risk of developing TB disease, which increases in varying states of immunodeficiency up to a $16 \%$ annual risk of activation of TB infection into TB disease in HIV patients [3]. In 2019, there were an estimated 10 million new incident cases of active TB disease worldwide [1]. Approximately two-thirds of 
all cases arise in eight countries alone, the vast majority of which have overwhelmed health services with limited resources [1]. This significant global burden of disease has been recognised by the World Health Organization (WHO) who launched the End TB initiative in 2016. Their aim is to reduce incidence, morbidity and mortality of this disease by improving diagnostic and therapeutic practices, as well as developing preventative strategies, through innovative research and education. By 2035, the goal is to reduce TB mortality by $95 \%$ and reduce overall incidence of TB by $90 \%$ worldwide [4]. Owing to the work of our predecessors, it has been estimated that 60 million lives have been saved globally in the 21st century so far [5].

Effective TB treatment is dependent on:

- $\quad$ Prompt diagnosis of TB and recognition of drug resistance;

- Promoting and ensuring patient adherence to regimens;

- Robust contact tracing and prophylactic treatment of contacts; and

- Screening for TB infection in high-risk groups.

There is ongoing extensive research into developing accurate, timely methods of detecting drug resistance, even in resource poor settings. Many effective, less toxic medications are under development. Furthermore, methods of promoting and ensuring drug adherence are being reviewed. In addition, there is vital research ongoing in proactive areas of TB prevention, such as screening for, and treatment of, TB infection and developing efficacious vaccines to halt the spread of this killer disease.

The aim of this article is to: review current practice in the diagnosis and treatment of TB; outline new diagnostic techniques under development; discuss new drug therapies and treatment regimens under review; and review the evidence for vaccination.

\section{Diagnosis}

Improving the efficiency and accuracy of TB diagnosis contributes to treatment efficacy. Pulmonary TB should be suspected when patients present with classical symptoms such as non-resolving cough, haemoptysis, fevers, night sweats and weight loss. Extrapulmonary TB, including TB lymphadenitis, TB meningitis, laryngeal TB, Pott's disease and abdominal TB, presents in a variety of manners. Special consideration should always be given to patients who have potential TB exposure, as well as immunocompromised patients who may present atypically. The diagnosis must be made by confirming the presence of the causative pathogen, $M t b$. A variety of methods are employed to confirm the diagnosis. In addition, it is essential that there is emphasis on early detection of potential drug resistance.

Drug resistance is a growing issue that threatens TB care worldwide. Traditionally this was categorised into rifampicin-resistant TB (RR-TB), multidrug-resistant TB (MDR-TB) or extensively drug-resistant TB (XDR-TB). MDR-TB is resistant to both rifampicin (RIF) and isoniazid (INH). Recently definitions have been updated to include pre-XDR-TB, which is TB that fulfils the definition for MDR-TB and RR-TB that is also resistant to any fluoroquinolone (FLQ). The updated definition for XDR-TB is strains that fulfil the definition for MDR-TB/RR-TB which are also resistant to any group A drug (namely levofloxacin (LFX), moxifloxacin (MFX), bedaquiline (BDQ) and linezolid (LZD)) [6]. Replacing the old XDR-TB definition referencing second-line injectable drugs (SLID), it highlights the trend towards use of oral regimens comprising recently developed or repurposed drugs. Despite the importance of early recognition, only $61 \%$ of patients with a new diagnosis of bacteriologically confirmed TB disease in 2019 were tested for RIF resistance [1]. This is in part related to access to diagnostics in resource-limited settings. There are numerous methods currently available, and under development, to determine drug resistance. For these diagnostics to be beneficial on a global scale they need to provide timely, accurate, cost-effective results in centres where access to power, equipment and technical expertise remains limited.

\section{Culture}

Culture of $M t b$ in a suitable medium remains the gold standard diagnostic test. The specimen can be cultured in solid (e.g. Löwenstein-Jensen or Middlebrook 7H11) or liquid media (e.g. for use with the BACTEC Mycobacterium Growth Indicator Tube (MGIT) 960 system). Sensitivity, specificity, contamination rates and time to detection vary widely amongst both media, with the WHO advocating for dual use of systems where practical. The major benefit of the advent of liquid-based systems is the rapid time to detection, often reducing time to growth by half with a mean time to detection of 12.8 days compared with 25.1-25.5 days for the previously mentioned solid media [7]. However, sub-optimal laboratory facilities in resource-limited settings often restrict its practical use [8]. While culture is not recommended for use as a first-line test, it remains an important part of TB diagnostics where persistent culture positivity can predict likelihood of relapse [9]. 


\section{Direct microscopy}

Direct microscopy is a fast and inexpensive method to identify acid-fast bacilli (AFB), the majority of which are mycobacteria [10]. Traditionally, Ziehl-Neelsen (ZN) stain was applied and the sample termed "smear positive" or "smear negative", depending on the presence or absence of AFB. Efficacy is operator dependent, resulting in a broad range of sensitivities and specificities reported in international studies, 25.3-81.6\% and 83.4-99\%, respectively [11, 12]. It is even less sensitive in high-risk groups, such as patients with HIV, and children [1]. Methods to improve efficacy include use of mercury vapour fluorescence and light-emitting diode (LED) microscopy, which have largely replaced traditional ZN staining [13]. Education and quality assurance for laboratory technicians is one of the most useful ways to ensure accurate diagnosis, as direct microscopy often remains the only method of diagnosis available in resource-limited settings [14]. Similar to culture, direct microscopy remains an integral part of monitoring response to treatment, measuring infectiousness, and predicting likelihood of relapse in patients who are smear positive at diagnosis.

\section{Molecular tests}

Given the limitations of culture and direct microscopy, the WHO recommends a biomolecular test as the initial diagnostic test in a suspect patient [1]. Current molecular tests endorsed by WHO include: Xpert MTB/RIF and Xpert MTB/RIF Ultra assays (Cepheid, Sunnyvale, USA); loop-mediated isothermal amplification test (TB-LAMP; Eiken Chemical, Tokyo, Japan); Truenat MTB, MTB Plus and MTBRIF Dx tests (Molbio Diagnostics, Goa, India) and lateral flow urine lipoarabinomannan assay (LF-LAM; Alere Determine TB LAM Ag, Abbott, San Diego, USA).

The WHO currently recommends Xpert (MTB/RIF or MTB/RIF Ultra) or Truenat (MTB or MTB Plus) as the initial diagnostic test of choice in suspected pulmonary TB [1]. They are cartridge based nucleic acid amplification tests (NAAT) that detect the presence of TB DNA, as well as common mutations associated with RIF resistance along the $r p o B$ gene, within $2 \mathrm{~h}$ [15]. The Xpert MTB/RIF and Xpert MTB/RIF Ultra assays are also endorsed by the WHO for diagnosing extrapulmonary TB and TB in children [1]. When compared with culture diagnosis, the Xpert assays have demonstrated $89 \%$ sensitivity and 99\% specificity at diagnosing pulmonary TB in adults [16]. The Xpert MTB/RIF Ultra assay has a higher sensitivity but lower specificity than the Xpert MTB/RIF assay, owing to its inability to accurately differentiate between dormant and active TB DNA $[17,18]$. While recommended for use, it is important to remember these assays have reduced sensitivity in certain populations such as children and patients coinfected with HIV, as well as in extrapulmonary TB $[16,19]$. Moreover, this technology is expensive and requires laboratory facilities with continuous access to power. To overcome this obstacle in resource-limited settings, there are a number of smaller, battery-operated technologies in development. To date, the GeneXpert Omni (Omni; Cepheid) appears to be the most promising potential candidate for widespread use. In a real-world analysis, it has been shown to be a cost-effective method when used in peripheral healthcare settings [20]. It allows diagnosis to be at/near the point of care, and thus avoids further delays and costs associated with transporting samples to specialised centres.

As well as the Omni, Cepheid is also developing the Xpert MTB/XDR assay. It aims to also detect resistant to INH, FLQ, ethionamide (ETH) and SLID. Similar to other Xpert assays, it is a NAAT that detects 16 clinically relevant mutations associated with resistance in under $90 \mathrm{~min}$ [21]. When compared with phenotypic drug sensitivity testing (pDST), it has a 94\% sensitivity and $100 \%$ specificity at detecting drug resistance [21]. There are large scale multicentre clinical trials ongoing to establish its real-world efficacy as a follow-on test to current Xpert MTB/RIF and MTB/RIF Ultra assays, prior to consideration for WHO recommendation. This assay is of paramount importance as the early recognition of drug resistance is a prerequisite to shorter drug regimens, which will be discussed in further detail elsewhere in this review.

While most biomolecular tests are NAAT detecting the presence of Mtb DNA, the LF-LAM test detects a lipopolysaccharide present in mycobacterial cell walls. While not in use in most countries in the developed world, the LF-LAM assay has been recommended for use in HIV-coinfected patients. It is a urinary antigen test that is often employed in resource-limited settings, and is of particular benefit in cases where a sputum sample cannot be obtained. It has a $42 \%$ sensitivity in HIV patients with TB symptoms [22]. However, it cannot distinguish between mycobacterial species, and can cross react with other fungal diseases. As such, it is used as an initial test in peripheral primary care centres in areas of high TB endemicity only, to determine whether symptomatic patients with HIV should be referred for further confirmatory testing [23].

\section{Line probe assays}

Another method of molecular detection of $M t b$ resistance is line probe assay (LPA). Genotype MTBDRplus and Genotype MTBDRsl (Hain LifeScience GmbH, Nehren, Germany) are used for the 
detection of $M t b$ and its associated drug resistance. The WHO approved Genotype MTBDRplus employs a series of steps to detect $M t b$ and mutations in rpoB and $k a t G$, which confer RIF and INH resistance, respectively [24]. Additionally, it can detect the presence of inhA promoter genes that confer resistance to low dose INH, which are also typically associated with ETH and prothionamide resistance [25]. This in vitro test delivers results in $<6 \mathrm{~h}$ [26]. When compared with traditional culture-based drug sensitivity, it is $78.5 \%$ sensitive and $100 \%$ specific at detecting RIF and INH resistance [27]. The WHO endorsed Genotype MTBDRsl 2.0 assay can also detect resistance conferring mutations of FLQ (gyrA and gyrB) and SLID (rrs and eis) [28]. Reported sensitivity and specificity are 100\% and 98.9\% for FLQ, and 89.2\% and $98.5 \%$ for SLID [29]. Even more sensitive than NAAT at detecting FLQ resistance, this rapid test could allow for use of FLQ in patients that might otherwise have faced a lengthier regime that potentially required the interim use of SLID. However, these tests are not without limitations including low sensitivity for detecting ethambutol (ETM) and aminoglycoside resistance as demonstrated in a real-world analysis [30]. Similar in aim to the Xpert MTB/XDR assay, these LPAs provide prompt recognition of drug resistance, so patients can be started on the appropriate regimen and further drug resistance does not have an opportunity to develop while awaiting standard culture-based susceptibility results, nor are patients exposed to burdensome, longer drug regimens with higher potential for toxicity.

Whole genome sequencing (WGS)

While NAAT and LPA tests are rapid, accessible diagnostics, their efficacy at detecting drug resistance is hindered by the inability to detect clinically relevant mutations outside the rifampicin resistance determining region (RRDR) of the $r p o B$ gene [31]. While 95\% of resistant cases arise from mutations in this region, there have been a number of public health crises emerging from missed diagnosis of outbreaks that have arisen from mutations outside it [32]. One such example is the I491F mutation that has been responsible for an outbreak of MDR-TB in Eswatini and remains a grave public health concern [33]. Another limitation is the inability to differentiate silent mutations from those that hinder drug efficacy, thus delivering a higher rate of false positive resistance results [34]. WGS provides a comprehensive review of the entire $M t b$ genotype with a 96\% concordance for culture-based sensitivity testing [35]. It provides genotypic sensitivity to most drugs required for treatment of MDR-TB [36]. While full clarification on clinical correlation between genotypic and phenotypic sensitivities remains to be shown, progress has been made in assigning probability of pDST based on genotypic results [37]. Utility was initially limited in low-income countries by cost and requirement for robust facilities and technical expertise [38]. However, with ongoing technological advancements in the microfluidic approaches to TB diagnosis, WGS is likely to be available at point of care on a global basis [39]. For some countries, it remains an important tool not only in case diagnosis, but in formulating public health policy by assisting in tracing TB contact cases in outbreaks [40]. In the future, with improved knowledge of the genomics involved in TB resistance, WGS is likely to prove revolutionary in tailoring TB treatment to each individual patient based on the particular genome identified by the $M t b$ strain they have contracted.

\section{Culture-based drug sensitivity testing (DST)}

As previously mentioned, the major advantage of liquid culture is rapidity of growth, which has led to more widespread use of liquid broth-based methods such as the MGIT. BACTEC MGIT 960 is a fully automated system that delivers results within 2 weeks [41]. Culture-based DST remains the gold standard for determining drug resistance at present [1]. The two approaches currently in use are the critical concentration and minimum inhibitory concentration (MIC). Classically, critical concentration was defined as the lowest concentration of a drug that inhibits growth of $95 \%$ of $M t b$ strain present. Owing to ongoing research, these critical concentrations are regularly updated with a recent reduction in the critical concentration required to determine RIF resistance, allowing for greater concordance between genotypic and phenotypic sensitivity results [42]. Alternatively, the MIC method is defined as the lowest concentration of a drug that results in complete inhibition of visual growth of the Mtb strain in vitro. Following extensive work completed by national reference laboratories, and international discussion and agreement, a new reference MIC protocol has been set and validated by European consortia [43].

\section{Computer aided detection for chest radiographs}

Given the limitations, in terms of time, cost and infrastructure, to the above testing methods, it has become clear that there need to be affordable, accessible methods of screening available in high-burden areas to assist with risk stratification for allocating further testing. One such proposed method is the use of computer software to digitally interpret chest radiographs, and assign a score indicating the likelihood of TB. The most commonly studied software is CAD4TB, currently on version 6 . When compared with NAAT, CAD4TB has been shown to have $90-100 \%$ sensitivity, and $23-45 \%$ specificity at detecting TB disease [44]. It performs similarly to expert clinicians and radiologists, with similar pitfalls including disease obscured by musculoskeletal findings and differentiating old scarring from new disease. Its use is 
intended for high-burden areas, that may lack readily accessible radiological expertise on site to interpret chest radiographs in a timely fashion [45]. It may assist peripheral health centres to determine which patients require further molecular testing.

\section{Serum biomarkers}

Another potential method for triage testing is serum biomarkers. Devising an accurate biomarker that upholds sensitivity across different ethnicities, HIV status and site of TB has proven difficult. However, a nine protein biosignature has recently been discovered which appears to remain efficacious in all of these cohorts. Using fibrinogen, $\alpha_{2}$-macroglobulin, C-reactive protein, matrix metalloproteinase- 9 , transthyretin, complement factor $\mathrm{H}$, interferon- $\gamma$, interferon- $\gamma$ inducible protein-10 and tumour necrosis factor- $\alpha$ as a host biosignature demonstrated $92 \%$ sensitivity and $72 \%$ specificity for determining TB from other diseases [46]. If available on a commercial level, this serum assay could rapidly and effectively determine which patients warranted further testing. It is important to note that most of these biomarkers are markers of inflammation, and as such are widely variable amongst patients and their differing metabolic and disease states. Evaluating serum biomarkers as predictors of response to treatment, potential for relapse and predictors of TB infection versus active disease will be discussed elsewhere.

\section{Treatment}

Alongside research into obtaining accurate and timely diagnostics, there is tremendous work ongoing in developing safe, efficacious, tolerable treatment regimens. The goals of treatment are not only to eradicate disease, but to prevent long-term morbidity arising from either the disease itself or as an adverse effect of the drugs in use. Successful treatment of drug-sensitive TB (DS-TB) has been reported in 85\% of patients [1]. Efficacy in drug-resistant forms is lower at $57 \%$ and is likely multifactorial [1]. To reflect this, there has been a trend towards oral drug regimens, where possible, given research highlighting patient preference and cost-effectiveness of these drugs [47]. We need to deliver a regimen that will not only aid our global goal of TB eradication, but in a manner that reflects our patients' wishes, and in doing so, promotes their compliance.

\section{Current treatment}

The current medications approved for use in TB treatment, and their notable side-effects are summarised in table 1.

TABLE 1 Current medications in use for TB treatment and their notable side-effects

\begin{tabular}{|c|c|}
\hline Drug & Notable side-effects \\
\hline Rifampicin (RIF) & $\begin{array}{l}\text { Hepatotoxicity, nausea, dyspepsia, abdominal pain, rash, } \\
\text { CYP450 interactions }\end{array}$ \\
\hline Isoniazid (INH) & $\begin{array}{l}\text { Hepatotoxicity, peripheral neuropathy", optic neuritis, CYP450 } \\
\text { interactions }\end{array}$ \\
\hline Pyrazinamide (PZA) & GI disturbance, hepatotoxicity, gout \\
\hline Ethambutol (EMB) & Optic neuropathy \\
\hline Levofloxacin (LFX)/Moxifloxacin (MFX) & $\begin{array}{l}\text { QT prolongation, tendonitis, hypoglycaemia, psychiatric } \\
\text { disturbance }\end{array}$ \\
\hline Bedaquiline (BDQ) & QT prolongation, CYP450 interactions \\
\hline Linezolid (LZD) & $\begin{array}{l}\text { Myelosuppression, dysglycaemia, peripheral neuropathy }{ }^{\#} \text {, optic } \\
\text { neuropathy }\end{array}$ \\
\hline Clofazimine (CFZ) & $\begin{array}{l}\text { Hepatotoxicity, GI disturbance, neurological disturbance, QT } \\
\text { prolongation, altered skin pigmentation }\end{array}$ \\
\hline Cycloserine (CYS) & Psychiatric disturbance, peripheral neuropathy \\
\hline Delamanid (DLM) & QT prolongation, CYP450 interactions \\
\hline Aminoglycosides (AMK,CAP, STR) & Nephrotoxicity, ototoxicity \\
\hline Ethionamide (ETH)/Prothionamide (Pro) & $\begin{array}{l}\text { Psychiatric disturbance, peripheral neuropathy, hepatotoxicity, } \\
\text { hypothyroidism, dysglycaemia }\end{array}$ \\
\hline p-Aminosalicylic acid (PAS) & Hepatotoxicity, dysglycaemia, hypothyroidism \\
\hline $\begin{array}{l}\text { Amoxicillin-Clavulanate }{ }^{\uparrow} \text { with Meropenem or } \\
\text { Imipenem-Cilastatin }\end{array}$ & Diarrhoea, candidiasis \\
\hline
\end{tabular}


TABLE 2 Current ATS/CDC/ERS/IDSA consolidated guidelines on treating drug-resistant TB

RR-TB

As per MDR-TB

INH-resistant TB

RIF+PZA+ETM+FLQ for 6 months (can discontinue PZA after 2 months; FLQ only required in patients with extensive disease, i.e. cavitary or bilateral infiltrates)

MDR-TB

First line Levofloxacin or Moxifloxacin with all 4 of: Bedaquiline + Linezolid + Clofazimine ${ }^{\#}+$ Cycloserine $^{\#}$

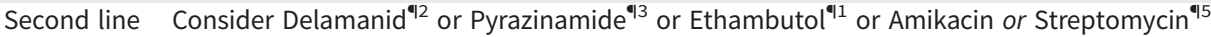

Third line Consider ETH ${ }^{\uparrow 6}$ or Prothionamide or Imipenem-Cilastatin/Clavulante or Meropenem/Clavulanate ${ }^{\uparrow 4}$ or p-Aminosalicylic Acid ${ }^{47}$ or High Dose Isoniazid

ATS: American Thoracic Society; CDC: US Centers For Disease Control And Prevention; ERS: European Respiratory Society; IDSA: Infectious Diseases Society of America. \#: in contrast, the WHO suggests only one of these drugs are required, comprising a 4-drug regimen (see text for full details); ${ }^{\uparrow}$ : superscript numbers refer to the order in which the WHO suggests drugs be incorporated into regimes. Information from [50].

DS-TB tends to follow a standard 6-month regime. This comprises an intensive phase with 2 months treatment consisting of RIF, INH, pyrazinamide (PZA) and ETM, followed by a continuation phase with 4 months treatment of RIF and INH [48]. If the isolate is susceptible to both RIF and INH, ETM can be stopped. The continuation phase should be extended to 7 months in the presence of: cavitation on the initial chest radiograph; persistent sputum growth at 2 months; or if PZA cannot be used due to monoresistance or drug side-effects. Consideration should also be given to extending this phase to 7 months in patients who are otherwise immunosuppressed, such as patients with HIV, diabetes mellitus, malignancy or medications associated with immunosuppression [48]. Unfavourable outcomes are most associated with high grade smear positivity (at least 3+) and dependent on the size of cavities, as well as extent of disease on chest radiographs [49].

Current treatment of drug-resistant TB is more complex and is summarised in table 2. Most notable is the longer duration of treatment involving combinations of drugs that are often poorly tolerated. There is also minor discordance between the two major international advisory bodies (the WHO and the joint ATS/ CDC/ERS/IDSA clinical practice guideline) concerning optimum drug selection and durations. While the WHO recommends only four drugs need to be used in the intensive phase of treatment, the ATS/CDC/ ERS/IDSA propose continuing to use five drugs in this phase. The ATS/CDC/ERS/IDSA have proposed this recommendation based on higher success rates in the five-drug group (93.9\% versus 89.7\%; adjusted odds ratio (aOR) 3.0 versus 1.2; risk difference $8 \%$ in both groups). Additionally, they suggest it is likely that one of the drugs may need to be withdrawn due to toxicity [50]. However, given equivocal risk differences in both groups, the WHO maintain four drugs should be sufficient, providing susceptibilities are known and toxicity is unlikely. De-escalation to a continuation phase comprising three or four drugs is based on similar evidence. Traditionally, MDR-TB required treatment for a total duration of 15-21 months [50]. Alternatively, it does allow for a shorter 9-12 month all oral regimen for patients who have not previously had more than 1 month of treatment with second-line medications, and in whom FLQ resistance has been ruled out. Additionally, patients should not have extensive disease [51]. This shorter regimen involves 4 months of six drugs (FLQ, clofazimine (CFZ), ETH, PZA, INH (high dose)), followed by 5 months of FLQ, CFZ, ETH and PZA. BDQ is used concurrently for the first 6 months of this regimen. This conditional recommendation of low certainty evidence was proposed owing to improved success and adherence rates, when compared with shorter regimens containing injectable agents (aOR 1.9 (95\% CI 1.6-2.4)) [52]. (Note, INH is used regardless of susceptibility status).

At present, the WHO recommends treatment for RR-TB in line with MDR-TB.

Pre-XDR- and XDR-TB are more difficult to treat, owing to varying patterns of drug resistance and advice should always be sought from national and international expert TB consortia prior to commencing treatment.

\section{New treatment: drugs}

At present, there are 16 new drugs in phase I or II clinical trials, and 22 other drugs in discovery or preclinical phases of development, as outlined in figure 1. Of those drugs undergoing clinical trial, there are 11 drugs of new chemical classes. Of the remaining drugs, TBAJ-587 and TBAJ-876 are diarylquinolines, similar to BDQ, while delpazolid, sutezolid and TBI-223 are oxazolidinones, similar to 


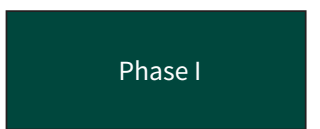

- BVL-GSK098

- GSK-286

- Macozinone

- TBAJ-587

- TBAJ-976

- TBI-166

- TBI-223

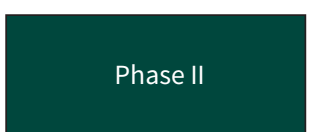

- BTZ-043

- Delpazolid

- GSK-656 (070)

- OPC-167832

-SPR720

- SQ-109

- Sutezolid

- TBA-7371

- Telacebec
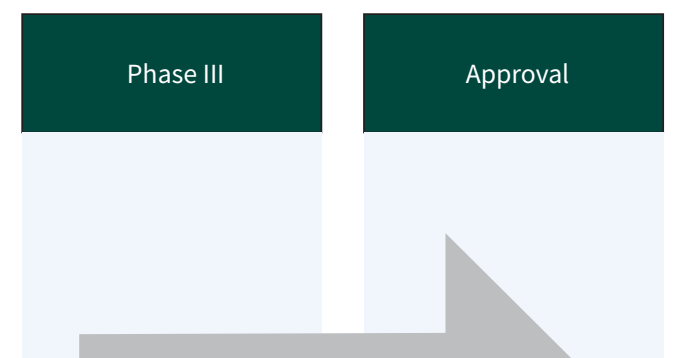

- Delamanid

- Bedaquiline

- Pretomanid

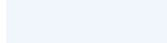

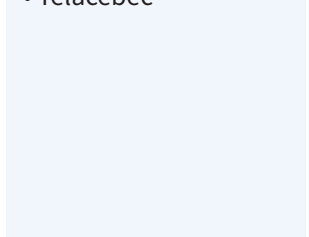

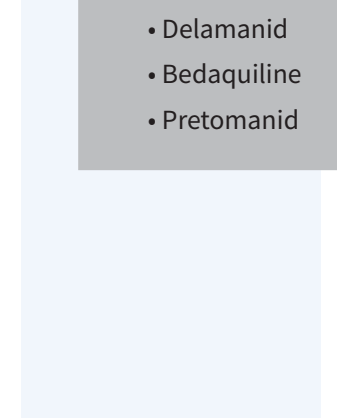

.

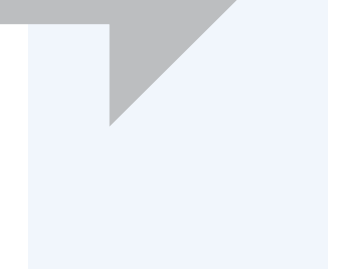

FIGURE 1 New anti-tuberculous drugs currently in development. Data from [104].

LZD and cycloserine. At the time of publication, no new drugs have reached phase III trials or been approved for market regulation since the approval of pretomanid $(\mathrm{Pa})$ in 2019. A promising candidate from a new drug class is telacebec. It induces bacterial cell death by inhibiting the mycobacterial cytochrome bc1 complex responsible for ATP synthesis. A proof-of-concept trial has shown increased rates of sputum clearance, with comparable levels of adverse events to currently approved drugs. If results from ongoing clinical trials continue to reflect this, it is likely to be approved as a third new modern drug class with anti-tuberculous activity [53]. This would be an important achievement as many of the other drugs in development are classified similarly to existing drugs, and as such their use in additive or substitutive places for their relative counterparts will be precluded due to concerns regarding toxicity or resistance. It is also interesting to note that these drugs in development are largely oral preparations, owing to patient preference and thus potential for greater adherence and cure.

\section{New treatment: routes of delivery}

While not a novel idea, interest in inhalation routes has been re-ignited. Numerous methods of drug delivery have been shown to be effective in animals, and additional advantages include reduced dosage and systemic toxicity. However, it would likely have no benefit on extrathoracic disease, nor would it be likely to achieve adequate therapeutic serum concentrations. Similar to the use of nebulised aminoglycosides in non-Mycobacterium tuberculosis, the potential for inhalational therapies to augment TB therapy likely lies as an adjunctive therapy to oral or injectable drug regimens. To date, there has been no data published from similar trials investigating its efficacy in sputum clearance from $M t b$ disease.

\section{New treatment: regimes}

In a disease that has the potential to affect one quarter of the world's population, it is astounding that no advances have been made in progressing the regimen for DS-TB since the mid-20th century. At present, international consensus guidelines continue to endorse standard 6-month regimes for the majority of cases of DS-TB, with varying longer regimens requiring expert opinion for drug-resistant cases. However, much research is being done into assessing shorter regimens with the aim of improving patient adherence and reducing risk of relapse and evolution of drug resistance, as seen in table 3.

1) DS-TB

- A shorter 4-month regimen of rifapentine (RFP) in combination with MFX has recently been shown to be non-inferior to the current standard 6-month regimen, as determined by negative smear or culture at 12 months, with no increase in major adverse events [54].

- The SimpliciTB group have evaluated a 4-month regimen comprising BDQ, Pa, MFX and PZA, in place of the standard 6-month regimen. While it has been said that no drug should ever be kept in reserve, it is unlikely that this regimen will be recommended as first-line therapy for DS-TB, given the need to preserve efficacious drug options for resistant cases [55, 56]. 
TABLE 3 New drug regimens under evaluation

\begin{tabular}{|c|c|c|c|c|}
\hline Trial & Drugs & Purpose & Status & Trial ID" \\
\hline ReDEFINe & RIF (HD) & $\begin{array}{l}\text { Assess high-dose RIF on treatment } \\
\text { outcomes and duration in TB } \\
\text { meningitis }\end{array}$ & Phase II & NCT02169882 \\
\hline TB-PRACTECAL & $\begin{array}{l}\text { BDQ, Pa, LZD, MFX, } \\
\quad \text { CFZ }\end{array}$ & $\begin{array}{l}\text { Assess } 6 \text { months of regimen for } \\
\text { MDR-TB }\end{array}$ & $\begin{array}{l}\text { Phase III } \\
\text { (data } \\
\text { Analysis) }\end{array}$ & NCT02589782 \\
\hline $\begin{array}{l}\text { Stream stage } \\
\quad 2\end{array}$ & $\begin{array}{l}\text { CFZ, ETM, MFX, PZA, } \\
\text { INH, Kan, Pro, } \\
\text { BDQ, LFX }\end{array}$ & $\begin{array}{l}\text { Varying combinations at differing } \\
\text { durations for RR-TB and MDR-TB }\end{array}$ & Phase III & NCT02409290 \\
\hline SimpliciTB & $\mathrm{BDQ}, \mathrm{Pa}, \mathrm{MFX}, \mathrm{PZA}$ & $\begin{array}{l}4 \text { months for DS-TB } \\
6 \text { months for MDR-TB }\end{array}$ & Phase III & NCT03338621 \\
\hline Truncate-TB & BDQ, RFP, LZD, MFX & 2 months for DS TB & Phase III & NCT03474198 \\
\hline TBTC study 31 & $\begin{array}{l}\text { RFP, INH, ETM, PZA, } \\
\quad \text { MFX }\end{array}$ & $\begin{array}{l}\text { Evaluate } 4 \text { months treatment for } \\
\text { DS-TB }\end{array}$ & Phase III & NCT02410772 \\
\hline endTB & $\begin{array}{l}\text { BDQ, DLM, LZD, } \\
\text { MFX, PZA, LFX, } \\
\text { CFZ }\end{array}$ & $\begin{array}{l}47 \text { weeks treatment with } \\
\text { combinations of } 5 \text { drugs for } \\
\text { MDR-TB }\end{array}$ & Phase III & NCT02754765 \\
\hline RIFASHORT & RIF (HD) & Evaluate 4 months of RIF (HD) & Phase III & NCT02581527 \\
\hline NEXT trial & $\begin{array}{l}\text { LZD, BDQ, PZA, ETH } \\
\quad \text { or INH (HD) }\end{array}$ & $\begin{array}{l}\text { Evaluate efficacy of ETH or INH (HD) } \\
\text { with 6-9 months treatment for } \\
\text { MDR-TB }\end{array}$ & $\begin{array}{l}\text { Phase III } \\
\text { (data } \\
\text { Analysis) }\end{array}$ & NCT02454205 \\
\hline ZeNix & $\mathrm{Pa}, \mathrm{LZD}, \mathrm{BDQ}, \mathrm{Pl}$ & Evaluate 26 weeks treatment & Phase III & NCT03086486 \\
\hline DELIBERATE & BDQ, DLM & Evaluate safety in MDR-TB & Phase III & NCT02583048 \\
\hline BEAT TB & $\begin{array}{l}\text { BDQ, DLM, LZD, LFX, } \\
\text { CFZ }\end{array}$ & $\begin{array}{l}\text { Evaluate safety and efficacy of } \\
6 \text { months treatment for RR-TB }\end{array}$ & Phase III & NCT04062201 \\
\hline
\end{tabular}

HD: high dose; RIF: rifampicin; INH: isoniazid; RFP: rifapentine; ETM: ethambutol; PZA: pyrazinamide; MFX: moxifloxacin; LFX: levofloxacin; LZD: linezolid; ETH: ethionamide; BDQ: bedaquiline; DLM: delamanid; Pa: pretomanid; CFZ: clofazamine; Kan: kanamycin; Pro: prothionamide; Pl: Placebo. \#: ClinicalTrials.gov identifier. Information from [1, 104].

- Shorter again with a 2-month regime, the TRUNCATE-TB trial is at recruitment phase. This multi-armed approach will assess combinations of 4-5 currently approved oral anti-tuberculous medications given daily for 8 weeks, with the potential to extend to 12 weeks [57].

- The RIFASHORT and ReDEFINe studies are evaluating the risk-benefit ratio of higher doses of RIF in DS-TB [58, 59]. The evidence base for these ongoing trials has been provided by the HIRIF trial which found an increased rate of sputum clearance, with no associated increase in toxicity, in patients on higher doses of RIF than currently recommended by the WHO [60].

2) Drug-resistant TB

a) RR-TB

- The current recommendations for RR-TB have been considered contentious for quite some time. These longer regimens likely expose patients with mono-resistance to unnecessarily long and toxic drug regimens, and also exclude the benefits of INH therapy [61]. BEAT TB is at the enrolment stage assessing the efficacy of 6 months of BDQ, LZD, delamanid (DLM), LFX and CFZ in comparison to current practices in South Africa [62].

- The updated STREAM2 study is evaluating a shorter regimen for RR-TB and MDR-TB in a simultaneous multi-armed approach. Their four regimens are based on: current WHO practice; the Bangladesh regime; a 40-week all oral regimen; and a 28-week oral regimen after an 8 week intense regime that also involves INH and kanamycin (Kan) [63, 64].

b) MDR-TB

- Results from the NEXT trial completed in December 2020 are awaited. This group compared 6-9 months of LZD, BDQ, LFX, PZA and ETH or INH (high dose) to current standards of care [65].

- TB-PRACTECAL stopped early due to superior outcomes in the intervention arm, consisting of a 6-month regimen of BDQ, Pa, LZD and MFX. Full results are awaited [66, 67].

- SimpliciTB are also evaluating a regimen for RR-TB/MDR-TB consisting of the same drugs as the DS-TB protocol (BDQ, PZA, MFX, Pa) but for 6 months [56].

- DELIBERATE are completing a phase II safety trial reviewing the safety and pharmacokinetics of combined BDQ and DLM therapy. Given the updated consensus guidelines, these drugs will often be 
given together and it is essential we have an evidence base for potential harms that may arise throughout the course of treatment [68].

- endTB, run by Médecins Sans Frontières, are evaluating a multi-armed approach combining varying combinations of an all oral regimen for 39 weeks. Similar to the STREAM2 study, this is the only other multi-armed trial reviewing multiple combinations of novel drugs simultaneously [69].

c) XDR-TB

- The ZeNix trial is the only trial, at present, that is reviewing treatment regimens for patients with pre-XDR- or XDR-TB. Using BDQ, Pa and either LZD (BPaL) or placebo for a total duration of 26 weeks, their aim is to assess rates of sputum conversion. This trial is also one of the few to follow patients for a significant period post-treatment, and patients with be reviewed for 78 weeks following the end of treatment [70]. Data from its predecessor the Nix-TB trial has shown $88 \%$ favourable outcomes at 24 months following treatment in patients with either MDR- or XDR-TB [71]. This $\mathrm{BPaL}$ regime can currently be used under operational research conditions in patients with MDR-TB, in accordance with WHO guidance [51].

While many of these trials demonstrate promise for an improved approach to TB treatment, it is essential that we see long-term data on their efficacy and relapse rates prior to implementing them on a global scale. The fear is that these patients may have excellent short-term results, but disease recurs soon after with the added potential for drug resistance to develop.

\section{New treatment: adjuncts}

In addition to shorter regimens, with new or re-purposed drugs, there is research into methods of modifying the host immune response to improve treatment outcomes and prevent permanent morbidity from TB disease. As previously discussed, upon infection with Mtb the host can either suppress bacillary replication into a latent state, or the host is overwhelmed and active disease develops [72]. Both deficient and hyperinflammatory states have been associated with TB disease morbidity and mortality, suggesting that tailoring a balanced immune response is of paramount importance to survival [73]. With evolving knowledge of the pathways and subcellular responses involved, new therapeutic targets are being developed to assist with bacillary quiescence in the so called "host directed therapy" approach [74]. Numerous drug targets have been suggested, largely centred on modulating macrophage activity [75]. Proposed adjunctive therapies include vitamin D, everolimus, auranofin and CC-11050, a novel anti-inflammatory compound. Preliminary results from trial data suggest none of these compounds improve rates of sputum conversion; however, patients in receipt of CC-11050 or everolimus had increased recovery of $\mathrm{FEV}_{1}$ (forced expiratory volume in $1 \mathrm{~s}$ ) post-treatment, perhaps solidifying the role of a balanced immune response to infection [76].

\section{New treatment: the future}

Going forward, with a combination of new drugs, altered durations and more effective testing of response to treatment, it is likely that each patient will have a tailored approach to TB treatment [49]. With studies like PredictTB, aiming to determine biomarkers and radiographic appearances that predict response and likelihood of relapse, we will be able to devise a drug combination and duration with greater specificity for each patient [77]. Similar technology may even assist with developing even more efficacious drugs in early-stage clinical trials [78]. Additionally, it is essential that any new drug or technology developed is affordable and available to all institutions, most importantly hospitals in low-resource environments, where the majority of the global TB burden persists.

\section{Adherence}

Despite ongoing research, treatment for DS-TB has remained unchanged for decades. This highly effective regimen is often poorly tolerated by patients, and "drug holidays" are frequent during treatment. This, of course, increases the likelihood of relapse and evolution of drug resistance. Moreover, patients with resistant TB have to endure longer regimens with their own associated side-effects. While awaiting the development and approval of less toxic regimens, there are a number of measures we can take to ameliorate adverse effects of treatment and promote patient adherence. It has been shown that comprehensive patient-centred approaches, involving nutritional, financial and psychological support, have higher rates of completion. In addition, patients with increased contact with healthcare workers tended to have lower drop-out rates during treatment [79]. The evidence base for this is provided by systematic reviews of mostly observational case studies and case cohorts, and as such randomised research in this area is required to determine a formal link. 


\section{Directly observed therapy}

Directly observed therapy (DOT) has been a standard of care in TB treatment for several years. The premise is that patients are more likely to comply if medication ingestion is witnessed multiple times per week. Current recommendations are that it should be implemented in MDR- or XDR-TB cases, or for patients with complex or vulnerable care needs, such as homelessness, comorbid psychiatric illness or addiction [80]. There have been conflicting results from systematic reviews on the efficacy of DOT [81, 82]. What is known, is that community-based DOT appears to be the most effective strategy, as it is less disruptive for patients and thus their adherence is more likely to be maintained [83]. In recent years, attention has switched towards the use of smartphone technology. Video observed therapy (VOT) has been suggested as an even less disruptive form of monitoring adherence [84]. Patients can either upload videos of medication ingestion to a secure platform to be watched at a later date, or it can be taken while on a live feed with their healthcare team. VOT has been shown to have a higher uptake rate and patient preference rating [85]. While plausible that this will improve adherence, and thus relapse should be less likely, this study was not sufficiently powered to assess this, nor did it follow up on relapse rates at an appropriate interval. A real-world efficacy and cost-effectiveness study is ongoing in a tertiary hospital in Ireland at present [86].

\section{Prophylaxis}

Undoubtedly, a burden of TB infection will persist for years to come. However, we have a chance to prevent many of these patients from progressing to active disease. Screening for TB infection in groups at high risk of progressing to TB disease remains a cost-effective and essential component to the global initiative. Screening via either of the endorsed interferon- $\gamma$ release assays (QuantiFERON-TB Gold In-Tube and T-SPOT.TB) or traditional tuberculin skin testing is recommended in certain populations. The WHO has advised that clinical judgement is paramount in interpreting these tests, and cautions that a higher rate of false negatives occurs in the most vulnerable populations [87]. Another essential component of the sustainable development goals is robust public health policy to assist in contact tracing of index cases and early treatment of contacts. In addition, prior to any prophylactic treatment being commenced, it is essential that due caution is taken to rule out the presence of active TB disease.

Currently the WHO advocates for treatment with 4 months of RIF or 6-9 months of INH in cases where the index case is known to be drug sensitive [87]. A 3-month combination of RIF and INH is also approved, although rarely used due to potential toxicity. Additionally, weekly INH and RFP for 3 months has been shown to demonstrate equal efficacy and toxicity in comparison to 6 months of INH therapy, while higher levels of adherence were noted in the INH/RFP arm [88]. Moreover, a 1-month regimen of RFP/INH therapy was non-inferior to 9 months INH monotherapy in preventing TB in HIV-infected patients [89]. However, this regimen has yet to be endorsed by major international consortia.

The recommendations for TB contacts of DS-TB cases who demonstrate evidence of TB infection are as per those above. For contacts of MDR-TB cases, the current recommendation is for 6-12 months treatment with a FLQ with or without a second drug. If a FLQ cannot be used due to resistance in the index case, treatment with ETM and PZA is to be considered [87]. Regardless of the regimen in use, it is vital that strict adherence is maintained to ensure efficacy and prevent resistance.

At present, the decision to treat is based on the potential for progression to active disease based on similar case profiles. Going forward, we could vastly improve the cost efficacy of this intervention by being able to determine exactly which patients were going to progress to active TB disease or not. It had been hoped the answer would lie in serum transcriptional biomarkers and host response-based gene signatures [90, 91]. Recently, a four-protein biomarker panel has shown $67.3 \%$ sensitivity and $96.3 \%$ specificity at determining active from latent TB [92]. This subclinical phase of TB disease can be difficult to interpret due to its lower inflammatory profile and person specific confounding factors that influence our immune response. Recent results from transcriptomic studies have been disappointing overall, but may potentially suggest a role for these panels in symptomatic patients with known TB infection and their risk of progression to TB disease in an imminent 6-month period [93].

\section{Vaccination}

Given the current prevalence of TB infection, with the associated lifetime risk of progressing to active disease, it is paramount that we protect future generations from this burden by halting transmission entirely. With greater understanding of the cellular processes involved in Mtb susceptibility and pathogenesis, scientists have been able to identify various potential targets with a role in vaccination. Central to this is the cellular immune response, with a need to upregulate T-helper cell (Th)1, and downregulate Th2 and regulatory T-cell responses [94]. It appears that $M t b$ has also recognised the need to adapt to this hypo-inflammatory phenotype with more modern strains displaying shorter latency and higher virulence than previously seen [95]. 
Self- evaluation questions

1. What proportion of the world's population are estimated to have TB infection (i.e. demonstrate immunological evidence of prior TB exposure)?
a) $5 \%$
b) $10 \%$
c) $25 \%$
d) $50 \%$

2. Which of the following is a WHO recommended first-line test in the diagnosis of TB disease?

a) Culture

b) Biomolecular test (e.g. Xpert MTB/RIF or Truenat MTB)

c) Line probe assay (e.g. Genotype MTBDRplus)

d) Serum interferon- $\gamma$ release assay

3. Pre-XDR-TB is defined as TB that is resistant to rifampicin, isoniazid and what other drug(s)?

a) Linezolid

b) Second-line injectable drugs (e.g. amikacin)

c) Fluoroquinolones (e.g. moxifloxacin)

d) Bedaquiline

4. Which of the following regimens are not currently recommended by the WHO for preventive treatment of TB disease (i.e. treatment of TB infection)?

a) Rifampicin ( $10 \mathrm{mg} \cdot \mathrm{kg}^{-1}$ up to $600 \mathrm{mg}$ maximum) daily for 4 months

b) Isoniazid ( $5 \mathrm{mg} \cdot \mathrm{kg}^{-1}$ up to $300 \mathrm{mg}$ maximum) daily for $6-9$ months

c) Rifampicin (10 $\mathrm{mg} \cdot \mathrm{kg}^{-1}$ up to $600 \mathrm{mg}$ maximum) and Isoniazid ( $5 \mathrm{mg} \cdot \mathrm{kg}^{-1}$ up to $300 \mathrm{mg}$ maximum) daily for 3 months

d) Rifapentine (900 mg if weight $>50 \mathrm{~kg}$, adjusted if less) and Isoniazid ( $15 \mathrm{mg} \cdot \mathrm{kg}^{-1}$ up to $900 \mathrm{mg}$ maximum) weekly for 1 month

The only worldwide approved vaccine against TB remains bacillus Calmette-Guérin (BCG), effectively reducing the risk of severe childhood disease from $\mathrm{TB}$, with an $85 \%$ reduction in $\mathrm{TB}$ meningitis and miliary TB in those $<10$ years of age [96]. It has also been noted that infants innoculated with BCG have increased survival and lower rates of other childhood infections. This observation is likely secondary to BCG's ability to prime innate immunity through epigenetic modification of innate immune cells [97].

Vaccination can be categorised into preventive pre-exposure, preventive post-exposure or therapeutic [98]. Vaccines can alternatively be classified according to their biochemical forms: live attenuated, inactivated, protein subunit or recombinant [99]. With each of these forms, the aim is to target various cells or subcellular components of TB pathogenesis.

MTBVAC, a pre-exposure live attenuated vaccine, has shown promising results from preclinical trials with a higher protection against TB than BCG [100]. This live vaccine is based on a genetically modified mutant $M t b$ strain containing deletions in transcription factors important for $M t b$ growth in macrophages and subsequent virulence.

VPM1002, another live recombinant BCG vaccine, is undergoing phase III studies at present to evaluate its efficacy at not only preventing infection, but in preventing active disease in those already affected [101]. This vaccine can modify T-cell immune response and enhance Th1 immunity, important in TB disease pathogenesis.

Another promising post-exposure candidate is M72/AS01E, a subunit vaccine, that prevents pulmonary TB in adults already infected with $M t b$ in $54 \%$ of patients, and thus could be a potentially life-saving intervention for one quarter of the world's population [102]. Also known as Mtb72F this vaccine comprises two immunogenic proteins that promote T-cell proliferation and interferon- $\gamma$ release [103].

Further randomised control trials are warranted in a timely manner if the END TB strategy is to be achieved.

\section{Conclusion}

The future is bright for TB treatment. Never before has there been such a global effort to develop new technologies and treatment for TB patients. Combining these advancements, it is possible that we will base each patient's treatment on their own protein biosignatures in conjunction with the genomic expression of mutations in the Mtb strain they have been affected with. If we are to achieve our goal of global eradication of TB, it is essential that we continue to collaborate and share our expertise on an international 
scale to ensure each patient gets the appropriate treatment and support to overcome their TB diagnosis without significant morbidity.

Conflict of interest: None declared.

\section{References}

1 World Health Organisation. Global tuberculosis report 2020. Geneva, World Health Organisation, 2020.

2 World Health Organisation. WHO consolidated guidelines on tuberculosis: tuberculosis preventive treatment: Module 1: prevention. Geneva, World Health Organisation, 2020.

3 Selwyn PA, Hartel D, Lewis VA, et al. A prospective study of the risk of tuberculosis among intravenous drug users with human immunodeficiency virus infection. N Engl J Med 1989; 320: 545-550.

$4 \quad$ Uplekar M, Weil D, Lonnroth K, et al. WHO's new end TB strategy. Lancet 2015; 385: 1799-1801.

5 Centers for Disease Control and Prevention. World Tuberculosis Day. 2021. www.cdc.gov/tb/features/wtbd/ 2021WTBD_Feature.html Date last updated: 4 March 2021.

6 World Health Organisation. WHO announces updated definitions of extensively drug-resistant tuberculosis 2021. https://www.who.int/news/item/27-01-2021-who-announces-updated-definitions-of-extensively-drugresistant-tuberculosis Date last updated: 27 January 2021.

7 Asmar S, Drancourt M. Rapid culture-based diagnosis of pulmonary tuberculosis in developed and developing countries. Front Microbiol 2015; 6: 1184.

8 World Health Organisation. Policy framework for implementing new tuberculosis diagnostics. Geneva, World Health Organisation, 2010.

9 Horne DJ, Royce SE, Gooze L, et al. Sputum monitoring during tuberculosis treatment for predicting outcome: systematic review and meta-analysis. Lancet Infect Dis 2010; 10: 387-394.

10 Sulis G, Centis R, Sotgiu G, et al. Recent developments in the diagnosis and management of tuberculosis. NPJ Prim Care Respir Med 2016; 26: 16078.

11 Bhalla M, Sidiq Z, Sharma PP, et al. Performance of light-emitting diode fluorescence microscope for diagnosis of tuberculosis. Int J Mycobacteriol 2013; 2: 174-178.

12 Deng Y, Duan Y-F, Ga S-P, et al. Comparison of LAMP, GeneXpert, mycobacterial culture, smear microscopy, TSPOT.TB, TBAg/PHA ratio for diagnosis of pulmonary tuberculosis. Curr Med Sci 2021; 41: 1023-1028.

13 Singhal R, Myneedu VP. Microscopy as a diagnostic tool in pulmonary tuberculosis. Int J Mycobacteriol 2015; 4: $1-6$.

14 Gilpin C, Kim SJ, Lumb R, et al. Critical appraisal of current recommendations and practices for tuberculosis sputum smear microscopy. Int J Tuberc Lung Dis 2007; 11: 946-952.

15 Lawn SD, Mwaba P, Bates M, et al. Advances in tuberculosis diagnostics: the Xpert MTB/RIF assay and future prospects for a point-of-care test. Lancet Infect Dis 2013; 13: 349-361.

16 Steingart KR, Schiller I, Horne DJ, et al. Xpert ${ }^{\circledast}$ MTB/RIF assay for pulmonary tuberculosis and rifampicin resistance in adults. Cochrane Database Syst Rev 2014; 1: CD009593.

17 Dorman SE, Schumacher SG, Alland D, et al. Xpert MTB/RIF Ultra for detection of Mycobacterium tuberculosis and rifampicin resistance: a prospective multicentre diagnostic accuracy study. Lancet Infect Dis 2018; 18: 76-84.

18 Arend SM, van Soolingen D. Performance of Xpert MTB/RIF Ultra: a matter of dead or alive. Lancet Infect Dis 2018; 18: 8-10.

19 Park M, Kon OM. Use of Xpert MTB/RIF and Xpert Ultra in extrapulmonary tuberculosis. Expert Rev Anti Infect Ther 2021; 19: 65-77.

20 Sohn H, Kasaie P, Kendall E, et al. Informing decision-making for universal access to quality tuberculosis diagnosis in India: an economic-epidemiological model. BMC Med 2019; 17: 155.

21 Cao Y, Parmar H, Gaur RL, et al. Xpert MTB/XDR: a 10-color reflex assay suitable for point-of-care settings to detect isoniazid, fluoroquinolone, and second-line-injectable-drug resistance directly from Mycobacterium tuberculosis-positive sputum. J Clin Microbiol 2021; 59: e02314-20.

22 Bjerrum S, Schiller I, Dendukuri N, et al. Lateral flow urine lipoarabinomannan assay for detecting active tuberculosis in people living with HIV. Cochrane Database Syst Rev 2019; 10: CD011420.

23 Engel N, Mwaura M. User perspectives on LF-LAM for the diagnosis of active tuberculosis: results from qualitative research. In: Lateral flow urine lipoarabinomannan assay (LF-LAM) for the diagnosis of active tuberculosis in people living with HIV: policy update (2019). Geneva, World Health Organistaion, 2019. World Health Organisation. The use of molecular line probe assays for the detection of resistance to isoniazid and rifampicin: policy update. Geneva, World Health Organisation, 2016.

25 Muller B, Streicher EM, Hoek KG, et al. inhA promoter mutations: a gateway to extensively drug-resistant tuberculosis in South Africa? Int J Tuberc Lung Dis 2011; 15: 344-351.

26 Somoskovi A, Dormandy J, Rivenburg J, et al. Direct comparison of the genotype MTBC and genomic deletion assays in terms of ability to distinguish between members of the Mycobacterium tuberculosis complex in clinical isolates and in clinical specimens. J Clin Microbiol 2008; 46: 1854-1857. 
Huang WL, Chen HY, Kuo YM, et al. Performance assessment of the GenoType MTBDRplus test and DNA sequencing in detection of multidrug-resistant Mycobacterium tuberculosis. J Clin Microbiol 2009; 47: 2520-2524.

HAIN LifeScience. Genotype MTBDRsIVER 2.0 instructions for use. Document IFA-317A-01. Nehren, HAIN LifeScience, 2015.

Gardee Y, Dreyer AW, Koornhof HJ, et al. Evaluation of the GenoType MTBDRsl Version 2.0 assay for second-line drug resistance detection of Mycobacterium tuberculosis isolates in South Africa. J Clin Microbiol 2017; 55: 791-800.

Javed H, Bakuła Z, Pleń M, et al. Evaluation of genotype MTBDRplus and MTBDRsl assays for rapid detection of drug resistance in extensively drug-resistant Mycobacterium tuberculosis isolates in Pakistan. Front Microbiol 2018; 9: 2265.

Siu GK, Zhang Y, Lau TC, et al. Mutations outside the rifampicin resistance-determining region associated with rifampicin resistance in Mycobacterium tuberculosis. J Antimicrob Chemother 2011; 66: 730-733.

Zaw MT, Emran NA, Lin Z. Mutations inside rifampicin-resistance determining region of rpoB gene associated with rifampicin-resistance in Mycobacterium tuberculosis. J Infect Public Health 2018; 11: 605-610.

Sanchez-Padilla E, Merker M, Beckert P, et al. Detection of drug-resistant tuberculosis by Xpert MTB/RIF in Swaziland. N Engl J Med 2015; 372: 1181-1182.

Alonso M, Palacios JJ, Herranz M, et al. Isolation of Mycobacterium tuberculosis strains with a silent mutation in rpoB leading to potential misassignment of resistance category. J Clin Microbiol 2011; 49: 2688-2690.

Nikolayevskyy V, Kranzer K, Niemann S, et al. Whole genome sequencing of Mycobacterium tuberculosis for detection of recent transmission and tracing outbreaks: A systematic review. Tuberculosis (Edinb) 2016; 98: 77-85.

Coll F, McNerney R, Preston MD, et al. Rapid determination of anti-tuberculosis drug resistance from whole-genome sequences. Genome Med 2015; 7: 51.

CRyPTIC Consortium and the 100,000 Genomes Project, Allix-Béguec C, Arandjelovic I, et al. Prediction of susceptibility to first-line tuberculosis drugs by DNA sequencing. N Engl J Med 2018; 379: 1403-1415.

Eddabra R, Ait Benhassou H. Rapid molecular assays for detection of tuberculosis. Pneumonia (Nathan) 2018; 10: 4

Molloy A, Harrison J, McGrath JS, et al. Microfluidics as a novel technique for tuberculosis: from diagnostics to drug discovery. Microorganisms 2021; 9: 2330.

Roycroft E, Fitzgibbon MM, Kelly DM, et al. The largest prison outbreak of TB in Western Europe investigated using whole-genome sequencing. Int J Tuberc Lung Dis 2021; 25: 491-497.

Tortoli E, Cichero P, Piersimoni C, et al. Use of BACTEC MGIT 960 for recovery of mycobacteria from clinical specimens: multicenter study. J Clin Microbiol 1999; 37: 3578-3582.

World Health Organisation. Technical Report on critical concentrations for drug susceptibility testing of isoniazid and the rifamycins (rifampicin, rifabutin and rifapentine). Geneva, World Health Organisation, 2021.

Schon T, Werngren J, Machado D, et al. Multicentre testing of the EUCAST broth microdilution reference method for MIC determination on Mycobacterium tuberculosis. Clin Microbiol Infect 2021; 27: 288 e1-288 e4.

Harris M, Qi A, Jeagal L, et al. A systematic review of the diagnostic accuracy of artificial intelligence-based computer programs to analyze chest x-rays for pulmonary tuberculosis. PLoS One 2019; 14: e0221339.

Murphy K, Habib SS, Zaidi SMA, et al. Computer aided detection of tuberculosis on chest radiographs: An evaluation of the CAD4TB v6 system. Sci Rep 2020; 10: 5492.

Morris TC, Hoggart CJ, Chegou NN, et al. Evaluation of host serum protein biomarkers of tuberculosis in sub-Saharan Africa. Front Immunol 2021; 12: 639174.

Migliori GB, Tiberi S, Zumla A, et al. MDR/XDR-TB management of patients and contacts: challenges facing the new decade. The 2020 clinical update by the Global Tuberculosis Network. Int J Infect Dis 2020; 92S: S15-S25.

48 Nahid P, Dorman SE, Alipanah N, et al. Official American Thoracic Society/Centers for Disease Control and Prevention/Infectious Diseases Society of America Clinical Practice Guidelines: Treatment of Drug-Susceptible Tuberculosis. Clin Infect Dis 2016; 63: e147-e195.

Imperial MZ, Nahid P, Phillips PPJ, et al. A patient-level pooled analysis of treatment-shortening regimens for drug-susceptible pulmonary tuberculosis. Nat Med 2018; 24: 1708-1715.

Nahid P, Mase SR, Migliori GB, et al. Treatment of drug-resistant tuberculosis. An official ATS/CDC/ERS/IDSA clinical practice guideline. Am J Respir Crit Care Med 2019; 200: e93-e142.

World Health Organisation. WHO consolidated guidelines on tuberculosis: Module 4: Treatment - Drug-resistant tuberculosis treatment. Geneva, World Health Organisation, 2020.

Mirzayev F, Viney K, Linh NN, et al. World Health Organization recommendations on the treatment of drug-resistant tuberculosis, 2020 update. Eur Respir J 2021; 57: 2003300. 
de Jager VR, Dawson R, van Niekerk C, et al. Telacebec (Q203), a new antituberculosis agent. N Engl J Med 2020; 382: 1280-1281.

Dorman SE, Nahid P, Kurbatova EV, et al. Four-month rifapentine regimens with or without moxifloxacin for tuberculosis. N Engl J Med 2021; 384: 1705-1718.

Prasad R, Gupta N, Banka A. Multidrug-resistant tuberculosis/rifampicin-resistant tuberculosis: Principles of management. Lung India 2018; 35: 78-81.

ClinicalTrials.Gov. Trial to evaluate the efficacy, safety and tolerability of BPaMZ in drug sensitive (DS-TB) adult patients and drug resistant (DR-TB) adult patients. https://clinicaltrials.gov/ct2/show/NCT03338621

ClinicalTrials.Gov. Two-month regimens using novel combinations to augment treatment effectiveness for drug-sensitive tuberculosis (TRUNCATE-TB). https://ClinicalTrials.gov/show/NCT03474198

ClinicalTrials.Gov. A randomised trial to evaluate toxicity and efficacy of $1200 \mathrm{mg}$ and $1800 \mathrm{mg}$ of rifampicin for pulmonary tuberculosis (RIFASHORT). https://clinicaltrials.gov/ct2/show/NCT02581527

ClinicalTrials.Gov. High-dose rifampicin for the treatment of tuberculous meningitis: a dose-finding study (ReDEFINe). https://clinicaltrials.gov/ct2/show/NCT02169882

Velasquez GE, Brooks MB, Coit JM, et al. Efficacy and safety of high-dose rifampin in pulmonary tuberculosis. a randomized controlled trial. Am J Respir Crit Care Med 2018; 198: 657-666.

Malenfant JH, Brewer TF. Rifampicin mono-resistant tuberculosis-a review of an uncommon but growing challenge for global tuberculosis control. Open Forum Infect Dis 2021; 8: ofab018.

ClinicalTrials.Gov. Building evidence for advancing new treatment for rifampicin resistant tuberculosis (RR-TB) comparing a short course of treatment (containing bedaquiline, delamanid and linezolid) with the current South African standard of care. https://clinicaltrials.gov/ct2/show/NCT04062201

ClinicalTrials.Gov. The evaluation of a standard treatment regimen of anti-tuberculosis drugs for patients with MDR-TB (STREAM). https://clinicaltrials.gov/ct2/show/NCT02409290

Van Deun A, Maug AK, Salim MA, et al. Short, highly effective, and inexpensive standardized treatment of multidrug-resistant tuberculosis. Am J Respir Crit Care Med 2010; 182: 684-692.

ClinicalTrials.Gov. An open-label RCT to evaluate a new treatment for patients with multi-drug resistant tuberculosis (NExT). https://clinicaltrials.gov/ct2/show/NCT02454205

ClinicalTrials.Gov. Pragmatic clinical trial for a more effective concise and less toxic MDR-TB treatment regimen(s) (TB-PRACTECAL). https://clinicaltrials.gov/ct2/show/NCT02589782

Medecins San Frontieres. Drug-resistant TB clinical trial ends enrolment early after positive initial data. https://msf.org.uk/article/drug-resistant-tb-clinical-trial-ends-enrolment-early-after-positive-initial-data Date last updated: 24 March 2021.

ClinicalTrials.Gov. Evaluating the safety, tolerability and pharmacokinetics of bedaqeuiline and delamanid, alone and in combination, for drug-resistant pulmonary tuberculosis. https://clinicaltrials.gov/ct2/show/ record/NCT02583048

ClinicalTrials.Gov. Evaluating newly approved drugs for multidrug-resistant TB (endTB). https://clinicaltrials. gov/ct2/show/NCT02754765.

ClinicalTrials.Gov. Safety and efficacy of various doses and treatment durations of linezolid plus bedaquiline and pretomanid in participants with pulmonary XDR-TB, Pre-XDR-TB or Non-responsive/Intolerant MDR-TB (ZeNix). https://clinicaltrials.gov/ct2/show/NCT03086486

Conradie F, Diacon A, Ngubane N, et al. Final results of the NIX-TB clinical study of BPaL regimen for highly resistant TB. In: Virtual CROI 2021 Conference on Retroviruses and Opportunistic Infections. 2021. www. croiconference.org/abstract/final-results-of-the-nix-tb-clinical-study-of-bpal-regimen-for-highly-resistant-tb/

Lee SH. Tuberculosis infection and latent tuberculosis. Tuberc Respir Dis (Seoul) 2016; 79: 201-206.

Tobin DM, Roca FJ, Oh SF, et al. Host genotype-specific therapies can optimize the inflammatory response to mycobacterial infections. Cell 2012; 148: 434-446.

Palucci I, Delogu G. Host directed therapies for tuberculosis: futures strategies for an ancient disease. Chemotherapy 2018; 63: 172-180.

Roca FJ, Whitworth LJ, Redmond S, et al. TNF induces pathogenic programmed macrophage necrosis in tuberculosis through a mitochondrial-lysosomal-endoplasmic reticulum circuit. Cell 2019; 178: 1344-1361. e11.

prospective, open-label, phase 2, randomised controlled trial. Lancet Respir Med 2021; 9: 897-908.

ClinicalTrials.Gov. Using biomarkers to predict TB treatment duration. https://clinicaltrials.gov/ct2/show/ NCT02821832

ClinicalTrials.Gov. NexGen EBA radiologic and immunologic biomarkers of sterilizing drug activity in tuberculosis. https://clinicaltrials.gov/ct2/show/NCT02371681

Law S, Daftary A, O'Donnell M, et al. Interventions to improve retention-in-care and treatment adherence among patients with drug-resistant tuberculosis: a systematic review. Eur Respir J 2019; 53: 1801030.

National Institute of Health and Care Excellence. NICE Guideline: Tuberculosis. 2016. https://www.nice.org. uk/guidance/ng33 Published: 13 January 2016. Date last updated: 12 September 2019 
Volmink J, Garner P. Directly observed therapy for treating tuberculosis. Cochrane Database Syst Rev 2007; 4: CD003343.

Tian JH, Lu ZX, Bachmann MO, et al. Effectiveness of directly observed treatment of tuberculosis: a systematic review of controlled studies. Int J Tuberc Lung Dis 2014; 18: 1092-1098.

Parums DV. Editorial: updates from the World Health Organization (WHO) on global treatment recommendations for drug-susceptible and multidrug-resistant tuberculosis. Med Sci Monit 2021; 27: e934292.

Story A, Garfein RS, Hayward A, et al. Monitoring therapy compliance of tuberculosis patients by using video-enabled electronic devices. Emerg Infect Dis 2016; 22: 538-540.

Story A, Aldridge RW, Smith CM, et al. Smartphone-enabled video-observed versus directly observed treatment for tuberculosis: a multicentre, analyst-blinded, randomised, controlled superiority trial. Lancet 2019; 393: 1216-1224.

Health Innovation Hub Ireland. St James' Hospital recruits patients with TB for Video Direct Observational Therapy study to investigate smartphone versus community care, in an Irish research first. https://hih.ie/ st-james-hospital-recruits-patients-with-tb-for-video-direct-observational-therapy-study-to-investigate-smart phone-versus-community-care-in-an-irish-research-first/ Date last accessed 11 September 2021. Date last updated: 29 October, 2020.

World Health Organisation. Latent tuberculosis infection: updated and consolidated guidelines for programmatic management. Geneva, World Health Organisation, 2018.

Sterling TR, Villarino ME, Borisov AS, et al. Three months of rifapentine and isoniazid for latent tuberculosis infection. N Engl J Med 2011; 365: 2155-2166.

Chaisson RE, Ramchandani R, Swindells S. One month of rifapentine plus isoniazid to prevent HIV-related tuberculosis. reply. N Engl J Med 2019; 381: e23.

Warsinske H, Vashisht R, Khatri P. Host-response-based gene signatures for tuberculosis diagnosis: a systematic comparison of 16 signatures. PLoS Med 2019; 16: e1002786.

Esmail H, Cobelens F, Goletti D. Transcriptional biomarkers for predicting development of tuberculosis: progress and clinical considerations. Eur Respir J 2020; 55: 1901957.

$\mathrm{Li} \mathrm{Z,} \mathrm{Hu} \mathrm{J,} \mathrm{Liu} \mathrm{P,} \mathrm{et} \mathrm{al.} \mathrm{Microarray-based} \mathrm{selection} \mathrm{of} \mathrm{a} \mathrm{serum} \mathrm{biomarker} \mathrm{panel} \mathrm{that} \mathrm{can} \mathrm{discriminate}$ between latent and active pulmonary TB. Sci Rep 2021; 11: 14516.

Scriba TJ, Fiore-Gartland A, Penn-Nicholson A, et al. Biomarker-guided tuberculosis preventive therapy (CORTIS): a randomised controlled trial. Lancet Infect Dis 2021; 21: 354-365.

Gong W, Liang Y, Wu X. The current status, challenges, and future developments of new tuberculosis vaccines. Hum Vaccin Immunother 2018; 14: 1697-1716.

Gagneux S. Host-pathogen coevolution in human tuberculosis. Philos Trans R Soc Lond B Biol Sci 2012; 367: 850-859.

Mangtani P, Abubakar I, Ariti C, et al. Protection by BCG vaccine against tuberculosis: a systematic review of randomized controlled trials. Clin Infect Dis 2014; 58: 470-480.

Kleinnijenhuis, J, Quintin J, Preijers F, et al. Bacille Calmette-Guerin induces NOD2-dependent nonspecific protection from reinfection via epigenetic reprogramming of monocytes. Proc Natl Acad Sci USA 2012; 109: 17537-17542.

8 Fatima S, Kumari A, Das G, et al. Tuberculosis vaccine: a journey from BCG to present. Life Sci 2020; 252: 117594.

Whitlow E, Mustafa AS, Hanif SNM. An overview of the development of new vaccines for tuberculosis. Vaccines (Basel) 2020; 8: 586.

0 Gonzalo-Asensio J, Marinova D, Martin C, et al. MTBVAC: attenuating the human pathogen of tuberculosis (TB) toward a promising vaccine against the TB epidemic. Front Immunol 2017; 8: 1803.

1 Nieuwenhuizen NE, Kulkarni PS, Shaligram U, et al. The recombinant Bacille Calmette-Guerin vaccine VPM1002: ready for clinical efficacy testing. Front Immunol 2017; 8: 1147.

Ottenhoff THM. A trial of M72/AS01E vaccine to prevent tuberculosis. N Engl J Med 2020; 382: 1576-1577.

Nabavinia MS, Naderi Nasab M, Meshkat Z, et al. Construction of an expression vector containing Mtb72F of Mycobacterium tuberculosis. Cell J 2012; 14: 61-66.

Working Group on New TB Drugs. Clinical Pipeline. www.newtbdrugs.org/pipeline/clinical Date last accessed 4 September 2021.

\section{Suggested answers}

1. c.

2. b.

3. c.

4. d. 